\title{
Influence of DAP seed priming and soaking durations on yield and yield associated traits in Okra
}

\author{
Gohar Shafi ${ }^{1}$, Neelam Ara ${ }^{1}$, Fahim Ullah Khan ${ }^{2,3, *}$, Yousaf Jamal ${ }^{4}$ and \\ Abdul Basir ${ }^{4}$ \\ 1Department of Horticulture, The University of Agriculture Peshawar-Pakistan \\ 2Department of Plant Breeding \& Genetics, The University of Agriculture, Peshawar-Pakistan \\ 3Barani Agricultural Research Station, Kohat-Pakistan \\ 4Department of Agriculture, The University of Swabi-Pakistan \\ *Corresponding author's email: fahimbiotech@gmail.com \\ Citation \\ Gohar Shafi, Neelam Ara, Fahim Ullah Khan, Yousaf Jamal and Abdul Basir. Influence of DAP seed priming and \\ soaking durations on yield and yield associated traits in Okra. Pure and Applied Biology. Vol. 4, Issue 3, 2015, pp \\ 389-397. http://dx.doi.org/10.19045/bspab.2015.43015
}

Received: 23/06/2015 Revised: 28/07/2015

Accepted: 04/08/2015

\section{Abstract}

An experiment was conducted at Ornamental Horticulture nursery, The University of Agriculture, Peshawar during July 2012 to study "Influence of DAP seed priming and soaking durations on yield and yield associated traits in okra". The experiment was laid down in Randomized Complete Block Design with split plot arrangement having three replications. Four DAP concentrations i.e. $0.5 \%, 1 \%, 1.5 \%, 2 \%$ with a difference of 4 hours soaking durations from 4 hours soaking to 48 hours along with un-primed seed were studied. Data were recorded on production traits viz. plant weight $(\mathrm{gm})$, number of pods plant ${ }^{-1}$, pod length $(\mathrm{cm})$, number of seeds pod $^{-1}$ and pod yield $\left(\mathrm{kg} \mathrm{ha}^{-1}\right)$. The results of the study revealed that all the studied traits were significantly affected by concentrations and soaking durations and its interactions. Mean values showed that maximum plant weight (489.12 gm), number of pods plant ${ }^{-1}$ (35.95), pod length $(11.09 \mathrm{~cm})$, number of seeds pod $^{-1}(53.26)$ pod yield $\left(3845.54 \mathrm{kgha}^{-1}\right)$ at $0.5 \%$ DAP solution. Mean values of interactions result showed that maximum number of pods plant ${ }^{-1}$ (47.16) and pod yield (5255.27 kg ha-1) was recorded by the seed soaked for 32hr in $0.5 \%$ DAP solution. Maximum number of pod length $(12.63 \mathrm{~cm})$ was recorded by the seed soaked for $24 \mathrm{hr}$ in $1 \%$ DAP solution, whereas, maximum number of seeds pod $^{-1}$ (66.13) was recorded by the seed soaked for $32 \mathrm{hr}$ in $1 \%$ DAP solution. DAP solution @ $0.5 \%$ performed best in most of the parameters while distilled water showed poor performance. DAP concentrations @ $0.5 \%$ for $32 \mathrm{~h}$ is recommended for seed priming for obtaining maximum yield in Okra.

Key words: Vegetable; Okra; Seed priming; DAP; Soaking duration; Yield.

\section{Introduction}

Okra (Abelmoschus esculentus L.) is a fast growing; heat-loving tropical annual crop found in the wild along the White Nile, belongs to the family Malvaceae. Okra originated in tropical Africa, was grown in the Mediterranean region, wild forms are also found in India. Okra is a popular summer crop. Flowers are cream colored and have the general shape and form of a 
hibiscus blossom except they do not splay out flat. Like other hibiscus blossoms the young tender pods are cooked in curries, stewed, and used in soups [1]. The crop grows well in hot weather, especially in the regions with warm nights $\left(>20 \mathrm{C}^{\circ}\right)$. It is widely grown in the plain areas of the country, particularly in the provinces of Punjab and Sindh, and is valued as a source of good income for the growers. In Pakistan, total area and production of okra is estimated to be 13.9 thousand hectares and 102.9 thousand tons, respectively during 2011-12.

The farmers reporting that they have primed seed in the past used it to gap-fill or in perceived dry years. In recent years, the use of priming has grown following participatory methods in India, Pakistan and Bangladesh. [2, 3]. Priming decreases the time span between the emergence of the first and the last seedlings [4].

Phosphorus plays an essential role in all physiological and biochemical processes in plants [5]. Seed priming in phosphorus solution is supposed to be an effective way of promoting early seedling growth in phosphorus-deficient soils and ensuring phosphorus supply through seed soaking [6, 7]. Even in soils with sufficiently high phosphorus content, a seed soaked in phosphorus can be beneficial due to improved availability of the nutrient [8]. The present experiment was therefore designed to determine the optimum DAP concentration, soaking duration and interactive effects as seed priming and its effects on yield and yield related traits of okra plant.

\section{Materials and methods}

The experiment on "influence of DAP seed priming and soaking durations on yield and yield associated traits in okra" was conducted at Ornamental Horticulture Nursery, The University of Agriculture,
Peshawar in July 2012. Seed of Okra (cv. Green Star) was used in this experiment.

Experimental Design and Treatments

The experiment was laid out in Randomized Complete Block (RCB) design with fifty two treatments each replicated thrice. The plot size was $0.5574 \mathrm{~m}^{2}\left(6 \mathrm{ft}^{2}\right)$. Row to row and plant to plant distances were 75 and 30 $\mathrm{cm}$ respectively. There were two factors studied in this experiment;

Factor A (DAP concentrations): The seeds soaked in different concentrations of DAP (Di Ammonium Phosphate).
1. $0.5 \%$
2. $1 \%$
3. $1.5 \%$
4. $2 \%$

Factor B (Soaking duration): Thirteen levels of soaking duration starting from zero till 48 hours with 4 hours intervals $(0,4,8,12,16,20,24,28,32,36,40,44,48)$.

\section{Soil Analysis}

Before sowing of seeds soil samples up to $25 \mathrm{~cm}$ depth were taken randomly from different parts of the field and were analyzed in the Soil Science Laboratory at The University of Agriculture, Peshawar for chemical characteristics.

Table A: Chemical characteristics of experimental field.

$\begin{array}{ll}\text { Electric conductivity } & 80.3 \mathrm{ds} \mathrm{m}^{-1} \\ \text { Organic matter } & 0.99 \% \\ \text { Nitrogen } & 0.81 \mathrm{mg} \mathrm{L}^{-1} \\ \mathrm{P}_{2} \mathrm{O}_{5} & 0.02 \mathrm{mg} \mathrm{L}^{-1} \\ \mathrm{~K}_{2} \mathrm{O} & 43.3 \mathrm{mg} \mathrm{L}^{-1} \\ \mathrm{pH} & 8.5\end{array}$

Preparation of field and method of sowing Before seed sowing the field was properly ploughed, and then leveled to have efficient distribution of irrigation water. Then well rotten Farm Yard Manure was added to the soil. $60 \mathrm{~cm}$ wide ridges were formed for each treatment in which 8 seeds were sown on its both sides keeping a distance of $60 \mathrm{~cm}$ $\mathrm{R}-\mathrm{R}$ and $30 \mathrm{~cm} \mathrm{P}-\mathrm{P}$ distance. The field was 
irrigated after sowing and the second irrigation was followed after a week.

\section{Preparation of DAP solution}

To prepare different DAP solution, DAP fertilizer weighing $0.5,1,1.5$ and $2 \mathrm{~g}$ was thoroughly mixed in $100 \mathrm{ml}$ distilled water using magnetic stirrer to make $0.5,1,1.5$ and $2 \%$ solution of DAP, respectively. All the solution was prepared in different beakers and was marked properly for further use.

\section{Method of seed priming}

Seed of Okra (cv. Green Star) was put in beaker containing respective DAP solutions for specific time. For example for $0.5 \%$ DAP solution for 12 hour, seeds were placed in the beaker containing $0.5 \%$ solution for 12 hours and were then in put in refrigerator till sowing time.

\section{Parameter studied}

Data on the following parameters were recorded during the course of experiment.

\section{Plant weight (gm)}

The data was recorded by weighing randomly selected 10 plants randomly in each plot with the help of electrical balance and then averages were calculated.

\section{Number of pod plant $^{-1}$}

The plants were selected at random basis in each plot and tagged. The total numbers of pods obtained from selected plants were counted and then the average of pods per plants was calculated.

\section{Pod length (cm)}

Average pod length was taken by measuring the pods picked from randomly selected plants using scale and their average was calculated.

\section{Number of seeds pod $^{-1}$}

For this purpose $10-15$ pods plant $^{-1}$ were selected. The seed were taken out with the help of fruit cutter, counted and their average was calculated.

\section{Pod yield (kg ha-1)}

Pod yield was worked out by the following formula:
Yield $\mathrm{kg} \mathrm{ha}{ }^{-1}=$ Yield plot $^{-1}(\mathrm{~kg}) /$ Area of plot $\left(\mathrm{m}^{2}\right)$ x $10000 \mathrm{~m}^{2}$

\section{Results and Discussion}

Plant weight (gm)

Statistical analysis of data showed that soaking of seeds with different concentration of phosphate solutions and various soaking durations had a significant effect on plant weight while their interaction had no significant effect on plant weight (Table 1). The mean values of experimental results revealed that maximum plant weight (489.12 gm) was recorded by the seed soaked in $0.5 \%$ phosphate solution which was statistical similar with plant weight (478.06 gm) when seeds were soaked in $1 \%$ phosphate solution while minimum plant weight (454.44 gm) was recorded by the seed soaked in $2 \%$ phosphate solution, while in case of various soaking duration maximum plant weight (543.09 gm) was recorded in seed soaked for $32 \mathrm{hr}$ followed by (532.14 gm) plant weight for seed soaked in $28 \mathrm{hr}$ while minimum (380.42 gm) plant weight was recorded for control seed plot. (Table 2). Viability and vigor of plant have profound influence on the establishment and yield of crop. Healthy plants with welldeveloped roots and shoots can more effectively mobilize nutrients and can better withstand adverse condition. The findings are in line with those of [6] who concluded that Zinc priming increases seedling fresh weight and height as it increases respiration and activities of amylase, lipase and glutamic pyruvic transaminase. Water soaked and nutrient primed maize seeds produced more seedlings weight as compared to dry seeds [9].

Number of Pods plant ${ }^{-1}$

Statistical analysis of data showed that soaking of seed in different concentration, various soaking durations and their interaction had significant effect on number of pods plant ${ }^{-1}$ (Table 1). The mean values of the experimental results shows that 
maximum number of pods plant ${ }^{-1}$ (35.95) was recorded by the seed soaked in $0.5 \%$ phosphate solution followed by (33.98) number of pods plant ${ }^{-1}$ in which seed were soaked in $1 \%$ phosphate solution while minimum number of pods plant ${ }^{-1}$ (29.87) was recorded in plot in which seed were soaked in $2 \%$ phosphate solution, while in case of various soaking duration maximum number of pods plant ${ }^{-1}$ (43.78) was recorded in seed soaked for $32 \mathrm{hr}$ followed by (41.49) number of pods plant $^{-1}$ for seed soaked in $28 \mathrm{hr}$ while minimum (21.11) number of pods plant ${ }^{-1}$ was recorded in plot in which seed were soaked for $48 \mathrm{hr}$. According to their interaction results maximum number of pods plant ${ }^{-1}$ (47.16) was recorded by the seed soaked for $32 \mathrm{hr}$ in $0.5 \%$ solution followed by (45.28) number of pods plant ${ }^{-1}$ in plot in which seed were soaked for $32 \mathrm{hr}$ in $1 \%$ phosphate solution while minimum number of pods plant ${ }^{-1}$ (19.85) was recorded in plot in which seed were soaked for $48 \mathrm{hr}$ in $1 \%$ phosphate solution. (Table 3). Priming increases yield parameters such as number of primary branches plant ${ }^{-1}$, number of pods plant ${ }^{-1}$ in raya [10]. The probable reason for increase in pods might be due to improved emergence and better seedling growth as endorsed by several researchers in wheat, rice, maize and chickpea $[2,3]$.

\section{Pod length (cm)}

Statistical analysis of data showed that soaking of seed in different concentrations, various soaking durations and their interaction had a significant effect on pod length (Table 1). Mean values of the experimental results maximum pod length $(11.09 \mathrm{~cm})$ was recorded by the seed soaked in $0.5 \%$ phosphate solution followed by $(11.05 \mathrm{~cm})$ pod length in which seed were soaked in $1 \%$ phosphate solution while minimum pod length $(10.38 \mathrm{~cm})$ was recorded in plot in which seed were soaked in $2 \%$ phosphate solution, while in case of various soaking duration maximum pod length $(11.98 \mathrm{~cm})$ was recorded in seed soaked for $16 \mathrm{hr}$ followed by $(11.86 \mathrm{~cm})$ pod length for seed soaked in $24 \mathrm{hr}$ while minimum $(9.36 \mathrm{~cm})$ pod length was recorded in plot in which seed were soaked for $48 \mathrm{hr}$. According to their interaction results maximum number of pod length $(12.63 \mathrm{~cm})$ was recorded by the seed soaked for $24 \mathrm{hr}$ in $1 \%$ solution followed by $(12.25$ $\mathrm{cm})$ pod length in plot in which seed were soaked for $28 \mathrm{hr}$ in $1.5 \%$ phosphate solution while minimum pod length $(8.60 \mathrm{~cm})$ was recorded in plot in which seed were soaked for $40 \mathrm{hr}$ in $1.5 \%$ phosphate solution (Table 4). Wheat seed primed in different $P$ concentration had significantly enhanced spike length $(\mathrm{cm})$. The reason could be the better seedling production in primed seed sown plots [11].

\section{Numbers of seed $\operatorname{~od}^{-1}$}

Statistical analysis of data showed that soaking of seed in different concentration, various soaking duration and their interaction had a significant effect on number of seeds $\operatorname{pod}^{-1}$ (Table 1). Mean values of the experimental results maximum number of seeds pod $^{-1}$ (53.26) was recorded by the seed soaked in $0.5 \%$ phosphate solution followed by (51.86) number of seeds pod $^{-1}$ in which seed were soaked in $1 \%$ phosphate solution while minimum number of seeds pod $^{-1}$ (46.17) was recorded in plot in which seed were soaked in $2 \%$ phosphate solution, while in case of various soaking duration maximum number of seeds $\operatorname{pod}^{-1}$ (63.53) was recorded in seed soaked for $32 \mathrm{hr}$ followed by (61.70) number of seeds pod $^{-1}$ for seed soaked in $28 \mathrm{hr}$ while minimum (33.81) number of seeds pod $^{-1}$ was recorded in plot in which seed were soaked for 48hr. According to their interaction results maximum number of seeds pod $^{-1}$ (66.13) was recorded by the seed soaked for $32 \mathrm{hr}$ in $1 \%$ solution followed by (65.72) number of seeds pod ${ }^{-1}$ in plot in which seed were soaked for $32 \mathrm{hr}$ in $0.5 \%$ phosphate 
Table 1. Mean squares for various yield traits as affected by priming of Okra with DAP concentrations at various soaking durations.

\begin{tabular}{|l|c|r|r|r|r|r|}
\hline \multicolumn{1}{|c|}{ SOV } & Df & Plant weight & Pods plant $^{-1}$ & Pod length & Seeds pod & Pod yield \\
\hline Replication & 2 & 538.25 & 21.98 & 1.76 & 35.29 & 46508.03 \\
\hline $\begin{array}{l}\text { Concentration } \\
\text { (C) }\end{array}$ & 3 & $10180.36^{* *}$ & $274.98^{* *}$ & $5.57 * *$ & $432.99^{* *}$ & $\begin{array}{r}2535083 . \\
1 *\end{array}$ \\
\hline $\begin{array}{l}\text { Soaking } \\
\text { duration (D) }\end{array}$ & 12 & $34425.47^{* *}$ & $570.37^{* *}$ & $10.01^{* *}$ & $1136.42^{* *}$ & $\begin{array}{r}6666365 . \\
9 * *\end{array}$ \\
\hline C x D & 36 & $159.57^{\mathrm{NS}}$ & $4.83^{*}$ & $1.73^{*}$ & $6.71^{* *}$ & $\begin{array}{r}36687.24 \\
*\end{array}$ \\
\hline Error & 103 & 169.61 & 3.07 & 0.42 & 2.03 & 6376.82 \\
\hline
\end{tabular}

Table 2. Plant weight (gm) as affected by priming of Okra with DAP concentrations at various soaking durations.

\begin{tabular}{|l|l|l|l|l|l|}
\hline \multirow{2}{*}{$\begin{array}{l}\text { Soaking } \\
\text { Durations }\end{array}$} & \multicolumn{5}{|c|}{ Concentration } \\
\cline { 2 - 6 } & $0.5 \%$ & $1 \%$ & $1.5 \%$ & $2 \%$ & Mean \\
\hline $0 \mathrm{~h}$ & 384.07 & 379.17 & 381.87 & 376.57 & $380.42 \mathrm{~J}$ \\
\hline $4 \mathrm{~h}$ & 432.87 & 419.43 & 405.53 & 402.23 & $415.02 \mathrm{H}$ \\
\hline $8 \mathrm{~h}$ & 470.27 & 458.07 & 422.27 & 417.93 & $442.13 \mathrm{~F}$ \\
\hline $12 \mathrm{~h}$ & 502.03 & 489.89 & 457.10 & 450.20 & $474.81 \mathrm{E}$ \\
\hline $16 \mathrm{~h}$ & 519.01 & 508.77 & 476.07 & 472.13 & $493.99 \mathrm{D}$ \\
\hline $20 \mathrm{~h}$ & 532.11 & 526.14 & 496.63 & 490.77 & $511.41 \mathrm{C}$ \\
\hline $24 \mathrm{~h}$ & 547.38 & 530.77 & 509.50 & 506.37 & $523.50 \mathrm{~B}$ \\
\hline $28 \mathrm{~h}$ & 544.50 & 536.47 & 527.03 & 520.57 & $532.14 \mathrm{~B}$ \\
\hline $32 \mathrm{~h}$ & 562.27 & 557.43 & 527.83 & 524.83 & $543.09 \mathrm{~A}$ \\
\hline $36 \mathrm{~h}$ & 521.03 & 508.07 & 497.63 & 492.27 & $504.75 \mathrm{C}$ \\
\hline $40 \mathrm{~h}$ & 485.20 & 469.10 & 457.07 & 451.47 & $465.71 \mathrm{E}$ \\
\hline $44 \mathrm{~h}$ & 448.63 & 432.60 & 423.10 & 416.20 & $430.13 \mathrm{G}$ \\
\hline $48 \mathrm{~h}$ & 409.23 & 398.87 & 391.63 & 386.17 & $396.48 \mathrm{I}$ \\
\hline Mean & $489.12 \mathrm{~A}$ & $478.06 \mathrm{~A}$ & $459.48 \mathrm{~B}$ & $454.44 \mathrm{~B}$ & \\
\hline
\end{tabular}

LSD value at $\mathrm{P} \leq 0.01$ for concentration $=18.23$

LSD value at $\mathrm{P} \leq 0.01$ for soaking durations $=10.55$

Means followed by the same letter are not significantly different using LSD test at $1 \%$ level of probability 
Table 3. Numbers of pods plant ${ }^{-1}$ as affected by priming of Okra with DAP concentrations at various soaking durations.

\begin{tabular}{|l|l|l|l|l|l|}
\hline \multirow{2}{*}{$\begin{array}{l}\text { Soaking } \\
\text { Durations }\end{array}$} & \multicolumn{5}{|c|}{ Concentration } \\
\cline { 2 - 6 } $0 \mathrm{~h}$ & $0.5 \%$ & $1 \%$ & \multicolumn{1}{|c|}{$1.5 \%$} & $2 \%$ & Mean \\
\hline $4 \mathrm{~h}$ & $24.21 \mathrm{wx}$ & $23.22 \mathrm{w}-\mathrm{z}$ & $23.07 \mathrm{w}-\mathrm{z}$ & $23.46 \mathrm{w}-\mathrm{z}$ & $23.49 \mathrm{I}$ \\
\hline $8 \mathrm{~h}$ & $32.56 \mathrm{o}-\mathrm{r}$ & $31.14 \mathrm{p}-\mathrm{t}$ & $28.44 \mathrm{tu}$ & $25.39 \mathrm{vw}$ & $29.38 \mathrm{G}$ \\
\hline $12 \mathrm{~h}$ & $33.53 \mathrm{~m}-\mathrm{p}$ & $32.01 \mathrm{o}-\mathrm{s}$ & $29.78 \mathrm{r}-\mathrm{u}$ & $27.33 \mathrm{uv}$ & $30.66 \mathrm{G}$ \\
\hline $16 \mathrm{~h}$ & $36.24 \mathrm{i}-\mathrm{m}$ & $34.01 \mathrm{mnno}$ & $31.87 \mathrm{o}-\mathrm{s}$ & $29.54 \mathrm{stu}$ & $32.91 \mathrm{~F}$ \\
\hline $20 \mathrm{~h}$ & $38.04 \mathrm{~g}-\mathrm{k}$ & $36.19 \mathrm{i}-\mathrm{m}$ & $32.94 \mathrm{n}-\mathrm{q}$ & $31.17 \mathrm{p}-\mathrm{t}$ & $34.58 \mathrm{E}$ \\
\hline $24 \mathrm{~h}$ & $41.13 \mathrm{def}$ & $38.44 \mathrm{f}-\mathrm{j}$ & $35.53 \mathrm{k}-\mathrm{n}$ & $34.18 \mathrm{mno}$ & $37.32 \mathrm{D}$ \\
\hline $28 \mathrm{~h}$ & $43.33 \mathrm{bcd}$ & $40.79 \mathrm{~d}-\mathrm{g}$ & $37.08 \mathrm{~h}-\mathrm{l}$ & $36.14 \mathrm{i}-\mathrm{m}$ & $39.34 \mathrm{C}$ \\
\hline $32 \mathrm{~h}$ & $44.54 \mathrm{abc}$ & $42.76 \mathrm{bcd}$ & $39.80 \mathrm{e}-\mathrm{h}$ & $38.86 \mathrm{f}-\mathrm{i}$ & $41.49 \mathrm{~B}$ \\
\hline $36 \mathrm{~h}$ & $47.16 \mathrm{a}$ & $45.28 \mathrm{ab}$ & $41.84 \mathrm{cde}$ & $40.82 \mathrm{~d}-\mathrm{g}$ & $43.78 \mathrm{~A}$ \\
\hline $40 \mathrm{~h}$ & $39.77 \mathrm{c}-\mathrm{h}$ & $37.85 \mathrm{~h}-\mathrm{k}$ & $35.77 \mathrm{j}-\mathrm{n}$ & $33.85 \mathrm{~m}-\mathrm{p}$ & $36.81 \mathrm{D}$ \\
\hline $44 \mathrm{~h}$ & $34.60 \mathrm{l}-\mathrm{o}$ & $32.51 \mathrm{o}-\mathrm{r}$ & $30.50 \mathrm{q}-\mathrm{t}$ & $24.14 \mathrm{wxy}$ & $30.44 \mathrm{G}$ \\
\hline $48 \mathrm{~h}$ & $29.76 \mathrm{r}-\mathrm{u}$ & $27.64 \mathrm{uv}$ & $23.80 \mathrm{wxy}$ & $22.64 \mathrm{w}-\mathrm{z}$ & $25.96 \mathrm{H}$ \\
\hline Mean & $22.47 \mathrm{xyz}$ & $19.85 \mathrm{z}$ & $21.37 \mathrm{yz}$ & $20.73 \mathrm{yz}$ & $21.11 \mathrm{~J}$ \\
\hline
\end{tabular}

LSD value at $\mathrm{P} \leq 0.01$ for different concentration $=1.801$

LSD value at $P \leq 0.01$ for various soaking durations $=1.419$

LSD value at $P \leq 0.05$ for various interactions $=2.838$

Means followed by the same letter are not significantly different using LSD test at 1 and $5 \%$ level of probability

Table 4. Pod length $(\mathrm{cm})$ as affected by priming of Okra with DAP concentrations at various soaking durations.

\begin{tabular}{|l|l|l|l|l|l|}
\hline \multirow{2}{*}{$\begin{array}{l}\text { Soaking } \\
\text { Durations }\end{array}$} & \multicolumn{5}{|c|}{ Concentration } \\
\cline { 2 - 6 } $0 \mathrm{~h}$ & $9.5 \%$ & $1 \%$ & $1.5 \%$ & $2 \%$ & Mean \\
\hline $4 \mathrm{~h}$ & $11.68 \mathrm{a}-\mathrm{i}$ & $11.01 \mathrm{f}-\mathrm{k}$ & $8.60 \mathrm{u}$ & $9.08 \mathrm{r}-\mathrm{u}$ & $10.09 \mathrm{EF}$ \\
\hline $8 \mathrm{~h}$ & $11.86 \mathrm{a}-\mathrm{g}$ & $11.05 \mathrm{f}-\mathrm{k}$ & $8.74 \mathrm{tu}$ & $9.78 \mathrm{n}-\mathrm{t}$ & $10.36 \mathrm{E}$ \\
\hline $12 \mathrm{~h}$ & $11.91 \mathrm{a}-\mathrm{f}$ & $11.69 \mathrm{a}-\mathrm{i}$ & $10.30 \mathrm{k}-\mathrm{q}$ & $10.65 \mathrm{i}-\mathrm{o}$ & $11.14 \mathrm{CD}$ \\
\hline $16 \mathrm{~h}$ & $12.47 \mathrm{abc}$ & $11.51 \mathrm{~b}-\mathrm{j}$ & $12.17 \mathrm{a}-\mathrm{e}$ & $11.77 \mathrm{a}-\mathrm{g}$ & $11.98 \mathrm{~A}$ \\
\hline $20 \mathrm{~h}$ & $12.51 \mathrm{ab}$ & $10.60 \mathrm{j}-\mathrm{p}$ & $11.85 \mathrm{a}-\mathrm{g}$ & $10.87 \mathrm{~g}-\mathrm{m}$ & $11.46 \mathrm{~A}-\mathrm{D}$ \\
\hline $24 \mathrm{~h}$ & $11.86 \mathrm{a}-\mathrm{g}$ & $12.63 \mathrm{a}$ & $11.73 \mathrm{a}-\mathrm{h}$ & $11.21 \mathrm{~d}-\mathrm{k}$ & $11.86 \mathrm{AB}$ \\
\hline $28 \mathrm{~h}$ & $11.58 \mathrm{a}-\mathrm{j}$ & $11.61 \mathrm{a}-\mathrm{j}$ & $12.25 \mathrm{a}-\mathrm{d}$ & $11.16 \mathrm{e}-\mathrm{k}$ & $11.65 \mathrm{ABC}$ \\
\hline $32 \mathrm{~h}$ & $10.64 \mathrm{i}-\mathrm{p}$ & $12.33 \mathrm{abc}$ & $11.05 \mathrm{f}-\mathrm{k}$ & $10.21 \mathrm{k}-\mathrm{q}$ & $11.06 \mathrm{D}$ \\
\hline $36 \mathrm{~h}$ & $11.21 \mathrm{~d}-\mathrm{k}$ & $11.42 \mathrm{c}-\mathrm{j}$ & $11.86 \mathrm{a}-\mathrm{g}$ & $10.99 \mathrm{f}-1$ & $11.37 \mathrm{BCD}$ \\
\hline $40 \mathrm{~h}$ & $10.25 \mathrm{k}-\mathrm{q}$ & $9.64 \mathrm{n}-\mathrm{u}$ & $8.60 \mathrm{u}$ & $9.89 \mathrm{~m}-\mathrm{s}$ & $9.59 \mathrm{FG}$ \\
\hline $44 \mathrm{~h}$ & $9.41 \mathrm{q}-\mathrm{u}$ & $10.68 \mathrm{~h}-\mathrm{n}$ & $9.95 \mathrm{l}-\mathrm{r}$ & $9.62 \mathrm{o}-\mathrm{u}$ & $9.91 \mathrm{EF}$ \\
\hline $48 \mathrm{~h}$ & $8.90 \mathrm{r}-\mathrm{u}$ & $8.85 \mathrm{stu}$ & $9.37 \mathrm{q}-\mathrm{u}$ & $10.32 \mathrm{k}-\mathrm{q}$ & $9.36 \mathrm{G}$ \\
\hline Mean & $11.09 \mathrm{~A}$ & $11.05 \mathrm{~A}$ & $10.47 \mathrm{~B}$ & $10.38 \mathrm{~B}$ & \\
\hline
\end{tabular}


Table 5. Numbers of seed pod $^{-1}$ as affected by priming of Okra with DAP concentrations at various soaking durations.

\begin{tabular}{|l|l|l|l|l|l|}
\hline \multirow{2}{*}{$\begin{array}{l}\text { Soaking } \\
\text { Durations }\end{array}$} & \multicolumn{5}{|c|}{ Concentration } \\
\cline { 2 - 6 } & $0.5 \%$ & $1 \%$ & \multicolumn{1}{|c|}{$1.5 \%$} & $2 \%$ & Mean \\
\hline $0 \mathrm{~h}$ & $39.77 \mathrm{vwx}$ & $38.90 \mathrm{vwx}$ & $37.17 \mathrm{wxy}$ & $34.25 \mathrm{yz}$ & $37.52 \mathrm{~J}$ \\
\hline $4 \mathrm{~h}$ & $50.93 \mathrm{pqr}$ & $49.96 \mathrm{qr}$ & $46.40 \mathrm{t}$ & $41.77 \mathrm{u}-\mathrm{x}$ & $47.27 \mathrm{H}$ \\
\hline $8 \mathrm{~h}$ & $54.47 \mathrm{klm}$ & $52.10 \mathrm{n}-\mathrm{q}$ & $47.35 \mathrm{st}$ & $46.53 \mathrm{t}$ & $50.11 \mathrm{G}$ \\
\hline $12 \mathrm{~h}$ & $56.60 \mathrm{jk}$ & $53.43 \mathrm{l}-\mathrm{o}$ & $51.22 \mathrm{o}-\mathrm{r}$ & $49.12 \mathrm{rs}$ & $52.59 \mathrm{~F}$ \\
\hline $16 \mathrm{~h}$ & $58.28 \mathrm{ij}$ & $56.59 \mathrm{jk}$ & $52.60 \mathrm{~m}-\mathrm{p}$ & $51.97 \mathrm{n}-\mathrm{q}$ & $54.86 \mathrm{E}$ \\
\hline $20 \mathrm{~h}$ & $62.92 \mathrm{c}-\mathrm{f}$ & $59.50 \mathrm{ghi}$ & $54.08 \mathrm{lmn}$ & $53.15 \mathrm{n}-\mathrm{p}$ & $57.41 \mathrm{D}$ \\
\hline $24 \mathrm{~h}$ & $63.42 \mathrm{bcd}$ & $61.83 \mathrm{def}$ & $58.47 \mathrm{hij}$ & $55.68 \mathrm{kl}$ & $59.85 \mathrm{C}$ \\
\hline $28 \mathrm{~h}$ & $64.58 \mathrm{abc}$ & $63.02 \mathrm{cde}$ & $60.65 \mathrm{fgh}$ & $58.53 \mathrm{hij}$ & $61.70 \mathrm{~B}$ \\
\hline $32 \mathrm{~h}$ & $65.72 \mathrm{ab}$ & $66.13 \mathrm{a}$ & $61.56 \mathrm{~d}-\mathrm{g}$ & $60.72 \mathrm{e}-\mathrm{h}$ & $63.53 \mathrm{~A}$ \\
\hline $36 \mathrm{~h}$ & $53.47 \mathrm{l}-\mathrm{o}$ & $52.81 \mathrm{~m}-\mathrm{p}$ & $43.71 \mathrm{t}-\mathrm{x}$ & $42.88 \mathrm{u}$ & $48.22 \mathrm{H}$ \\
\hline $40 \mathrm{~h}$ & $47.26 \mathrm{st}$ & $45.87 \mathrm{tu}$ & $38.67 \mathrm{vwx}$ & $37.55 \mathrm{wxy}$ & $42.34 \mathrm{I}$ \\
\hline $44 \mathrm{~h}$ & $40.88 \mathrm{u}-\mathrm{x}$ & $39.85 \mathrm{vwx}$ & $35.90 \mathrm{xyz}$ & $34.79 \mathrm{xyz}$ & $37.85 \mathrm{~J}$ \\
\hline $48 \mathrm{~h}$ & $34.05 \mathrm{yz}$ & $34.12 \mathrm{yz}$ & $33.77 \mathrm{yz}$ & $33.29 \mathrm{z}$ & $33.81 \mathrm{~K}$ \\
\hline Mean & $53.26 \mathrm{~A}$ & $51.86 \mathrm{~A}$ & $47.81 \mathrm{~B}$ & $46.17 \mathrm{~B}$ & \\
\hline
\end{tabular}

LSD value at $\mathrm{P} \leq 0.01$ for different concentration $=2.765$

LSD value at $P \leq 0.01$ for various soaking durations $=1.154$

LSD value at $P \leq 0.05$ for various interactions $=2.309$

Means followed by the same letter are not significantly different using LSD test at 1 and $5 \%$ level of probability

Table 6. Pod yield $\left(\mathrm{kg} \mathrm{ha}^{-1}\right)$ as affected by priming of Okra with DAP concentrations at various soaking durations.

\begin{tabular}{|l|l|l|l|l|l|}
\hline \multirow{2}{*}{$\begin{array}{l}\text { Soaking } \\
\text { Durations }\end{array}$} & \multicolumn{5}{|c|}{ Concentration } \\
\cline { 2 - 6 } $0 \mathrm{~h}$ & $2482.67 \mathrm{yz}$ & $2364.30 \mathrm{yz}$ & $2333.97 \mathrm{z}$ & $2445.80 \mathrm{yz}$ & $2406.68 \mathrm{~L}$ \\
\hline $4 \mathrm{~h}$ & $3095.27 \mathrm{rst}$ & $2988.67 \mathrm{tu}$ & $2787.73 \mathrm{wxy}$ & $2695.20 \mathrm{xy}$ & $2891.72 \mathrm{~J}$ \\
\hline $8 \mathrm{~h}$ & $3229.70 \mathrm{pq}$ & $3118.67 \mathrm{qrs}$ & $2920.53 \mathrm{uv}$ & $2750.23 \mathrm{xy}$ & $3004.78 \mathrm{I}$ \\
\hline $12 \mathrm{~h}$ & $3621.50 \mathrm{kl}$ & $3427.07 \mathrm{mn}$ & $3226.93 \mathrm{pq}$ & $3091.65 \mathrm{rst}$ & $3341.79 \mathrm{G}$ \\
\hline $16 \mathrm{~h}$ & $3902.73 \mathrm{~h}$ & $3725.17 \mathrm{ijk}$ & $3448.33 \mathrm{mn}$ & $3213.23 \mathrm{pqr}$ & $3572.37 \mathrm{H}$ \\
\hline $20 \mathrm{~h}$ & $4313.87 \mathrm{f}$ & $4066.63 \mathrm{~g}$ & $3827.73 \mathrm{hi}$ & $3511.401 \mathrm{~m}$ & $3929.91 \mathrm{D}$ \\
\hline $24 \mathrm{~h}$ & $4609.47 \mathrm{~d}$ & $4321.23 \mathrm{f}$ & $4080.10 \mathrm{~g}$ & $3834.80 \mathrm{hi}$ & $4211.40 \mathrm{C}$ \\
\hline $28 \mathrm{~h}$ & $4924.03 \mathrm{~b}$ & $4703.13 \mathrm{~cd}$ & $4382.93 \mathrm{ef}$ & $4070.57 \mathrm{~g}$ & $4520.17 \mathrm{~B}$ \\
\hline $32 \mathrm{~h}$ & $5255.27 \mathrm{a}$ & $5008.80 \mathrm{~b}$ & $4779.77 \mathrm{c}$ & $4479.78 \mathrm{e}$ & $4880.90 \mathrm{~A}$ \\
\hline $36 \mathrm{~h}$ & $4468.63 \mathrm{e}$ & $4380.07 \mathrm{ef}$ & $4073.57 \mathrm{~g}$ & $3717.63 \mathrm{ijk}$ & $4159.98 \mathrm{C}$ \\
\hline $40 \mathrm{~h}$ & $3878.47 \mathrm{~h}$ & $3788.63 \mathrm{hij}$ & $3682.10 \mathrm{jk}$ & $3363.23 \mathrm{no}$ & $3678.11 \mathrm{E}$ \\
\hline $44 \mathrm{~h}$ & $3296.67 \mathrm{op}$ & $3180.83 \mathrm{p}-\mathrm{s}$ & $3080.73 \mathrm{st}$ & $2844.90 \mathrm{vwx}$ & $3100.78 \mathrm{H}$ \\
\hline $48 \mathrm{~h}$ & $2913.70 \mathrm{uvw}$ & $2842.20 \mathrm{vwx}$ & $2803.60 \mathrm{v}-\mathrm{y}$ & $2281.20 \mathrm{z}$ & $2710.18 \mathrm{~K}$ \\
\hline Mean & $3845.54 \mathrm{~A}$ & $3685.80 \mathrm{~B}$ & $3494.46 \mathrm{C}$ & $3253.82 \mathrm{D}$ & \\
\hline
\end{tabular}


solution while minimum number of seeds $\operatorname{pod}^{-1}$ (33.29) was recorded in plot in which seed were soaked for $48 \mathrm{hr}$ in $2 \%$ phosphate solution (Table 5). Maximum pod length were recorded in plots receiving seeds soaked for $16 \mathrm{hrs}$, while seeds pod $^{-1}$ were maximum in plots received seeds soaked for $32 \mathrm{hrs}$, this might be due to production of long pods bearing less seeds or bold seeds. Probable reason could be that seed priming enhanced okra growth and resulted in more seeds pod $^{-1}$ compared to un-primed seeds. Previous investigation showed that seed soaking increased the number of spike $\mathrm{m}^{-2}$, number of grains spike ${ }^{-1}$ and grain yield compared with other treatments in wheat [12].

\section{Pod yield (ton ha ${ }^{-1}$ )}

Statistical analysis of data showed that soaking of seed in different concentration, various soaking durations and their interaction had a significant effect on pod yield $\left(\mathrm{kg} \mathrm{ha}^{-1}\right)$ (Table 1$)$. Mean values of the experimental results maximum pod yield (3.845 ton $\mathrm{ha}^{-1}$ ) was recorded by the seed soaked in $0.5 \%$ phosphate solution followed by (3.685 ton $\mathrm{ha}^{-1}$ ) pod yield in which seed were soaked in $1 \%$ phosphate solution while minimum pod yield (3.253 ton $\mathrm{ha}^{-1}$ ) was recorded in plot in which seed were soaked in $2 \%$ phosphate solution, while in case of various soaking duration maximum pod yield (4.880 ton $\mathrm{ha}^{-1}$ ) was recorded in seed soaked for $32 \mathrm{hr}$ followed by (4.520- ton ha ${ }^{1}$ ) pod yield for seed soaked in $28 \mathrm{hr}$ while minimum (2.406 ton ha ${ }^{-1}$ ) pod yield was recorded in un-primed seed plot. According to their interaction results maximum pod yield (5.255 ton $\mathrm{ha}^{-1}$ ) was recorded by the seed soaked for $32 \mathrm{hr}$ in $0.5 \%$ solution followed by (5.008- ton $\mathrm{ha}^{-1}$ ) pod yield in plot in which seed were soaked for $32 \mathrm{hr}$ in $1 \%$ phosphate solution while minimum pod yield (2.281- ton $\mathrm{ha}^{-1}$ ) was recorded in plot in which seed were soaked for $48 \mathrm{hr}$ in $2 \%$ phosphate solution (Table 6). The improved yield of primed seeds plots may be due to uniform and improved germination, vigorous seedling growth, well developed root system and efficient subsequent growth which ultimately led to higher yield [3]. Priming treatment significantly increased total biomass and plant weight as compared with control [13].

\section{Conclusion}

Seed priming with DAP solution produces more yield than un-primed seeds. Seed priming with $0.5 \%$ concentration solution up to $32 \mathrm{hr}$ perform better as compared to higher concentration soaking durations i.e. beyond 32 hr. Therefore, okra seed should be primed with DAP fertilizer solution before sowing for early germination, rapid seedling height and higher pod yield.

\section{References}

1. Baloch, AF (1990). Growth and yield performance of okra (Abelmoschus esculentus L.) cultivars. Gomal University J Res 10(2): 91-95.

2. Harris D, Joshi A, Khan PA, Gothkar P \& Sodhi PS (1999). On-farm seed priming in semi-arid agriculture: development and evaluation in maize, rice and chickpea in india using participatory methods. $J$ of Cambridge 35 (01): 15-29.

3. Harris D, Raghuwanshi BS, Ganwar JS, Singh SC, Joshi KD, Rashid A, Hollington PA (2001). Participatory evolution by farmers of on farm seed priming in wheat in India, Nepal, and Pakistan. Experimental Agri 3(7): 403-415.

4. Hil HJ (2004). Invigoration of Seed. Seed Science Technology. 3:881-888.

5. Hartmann TH, Geneve LR (2000). Plant propagation: principles and practices. Prentice hall, New Jersey. 8(8): 1014. 
6. Bieleski RL (1973). Phosphate pools, phosphate transport, and phosphate availability. Annual Review Plant Physio 24: 225-252.

7. Valdes AW (1987). Effect of seed coating and osmotic priming on the germination of seeds. Journal of American Soci of Horticulture Sci 11(2): 153-156.

8. Scott JM, Hill CB \& Jessop RS (1991). Growth chamber study of phosphorus applied as drilled granules or as seed coatings to wheat sown in soils differing in $\mathrm{p}$ absorption capacity. Fertilizer Res 29:281-287.

9. Arif M, Ali S, Shah A, Javeed N \& Rashid A (2005). Seed priming maize for improving emergence and seedling growth. Sarhad J of Agri 21(4): 539-543.

10. Ullah MA, Sarfraz M, Sadiq M, Mehdi SM \& Hassan G (2002). Effect of pre-sowing seed treatment of raya with micronutrients on yield parameters. Asian J of Plant Sci 1 (3): 277-78.

11. Shitab K \& Khalil SK (2007). Effect of seed priming with phosphorus concentrations and application rates on wheat. A thesis submitted to NWFP Agricultural University, Peshawar.

12. Ghosh DC, Mandal BP \& Malik GC (1997). Growth and yield of wheat (Triticum aestivum L.) as influenced by fertility level and seed-soaking agro-chemicals. Indian J of Agri Sci 67(4):144-146.

13. Basra SMA, Ehsanullah EA, Warraich MA, Cheema \& Afzal I (2003). Effect of storage on growth and yield of primed canola (Brassica napus L.) seeds. Intern $J$ of Agri and Biol 3(4):117-120. 